\title{
Characterisation of dendritic cell subsets in lung cancer micro-environments
}

\author{
A. Bergeron*,\#, F. El Hage*, M. Kambouchner*, D. Lecossier" and A. Tazi*,\#
}

\begin{abstract}
The aim of the current study was to seek evidence for a correlation between mediators present in lung cancer micro-environments and subsets of dendritic cells (DCs) infiltrating these tumours.

Immunohistochemistry and recently available antibodies were used to define the phenotype of DCs present in surgical biopsies from 12 patients with lung carcinomas, and the local expression of chemokines potentially involved in the recruitment of these cells was evaluated, both at mRNA and protein levels. Real-time PCR was used to analyse the expression of mRNA coding for cytokines known to influence the maturation of DCs in vitro.

Different subsets of myeloid DCs were present in lung cancers, but no plasmocytoid DCs were identified. Both Langerhans cells and CD1a+/Langerin cells were interspersed among tumour cells, in numbers that were correlated to the amounts of $C C$ chemokine ligand 20 produced in these tumours. In most specimens, DC-specific intercellular adhesion molecule-grabbing nonintegrin-postive DCs were also present at the periphery of the tumour beds. No DC-lysosomal associated membrane protein-positive DCs were identified and CD83+ DCs were rarely present in the tumour stroma. All tumours expressed interleukin (IL)-10, transforming growth factor- $\beta$ and vascular endothelial growth factor, whereas IL-12 was virtually absent.
\end{abstract}

Thus, various types of dendritic cells infiltrate lung carcinomas and display an immature phenotype, presumably because of the inhibitory cytokine micro-environment.

\section{KEYWORDS: Chemokines, cytokines, immunohistochemistry, Langerhans cells, PCR}

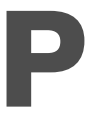

romising results have been previously reported using dendritic cell (DC)-based vaccination against particularly immunogenic malignant tumours such as metastatic melanomas and renal carcinomas [1, 2]. Subsequently, numerous clinical trials have evaluated various strategies for using DCs in immunotherapy for patients with advanced cancers, including lung cancer [3-5]. Although a T-cellmediated immune response has been repeatedly shown in these patients, the clinical effectiveness of these therapeutic approaches was generally limited [3]. Thus, questions have been raised concerning the type and activation state of human DCs that should be used to induce an effective antitumoural immunity.

Indeed, DCs are highly heterogeneous cell populations, and numerous subsets of DCs with different functional capacities have been identified $[3,6]$. Thus, myeloid DCs can be derived in vitro from CD34+ precursors or from blood monocytes, and these DCs can differentiate into Langerhans cells (LCs), particularly in the presence of transforming growth factor (TGF)- $\beta[3$, 6]. Plasmacytoid DCs are derived from lymphoid precursors, express the interleukin (IL)-3 receptor (R; CD123) at their surface and are dependent for their growth on the presence of IL-3 [3, 6]. DCs are also functionally heterogeneous according to their state of maturation $[3,6]$. Thus, immature DCs (such as myeloid DCs normally present in peripheral tissues and LCs in the epidermis or the respiratory tract mucosa) capture antigens but weakly stimulate $\mathrm{T}$ lymphocytes. In the presence of particular signals, such as lipopolysaccharide (LPS) or various cytokines, these cells mature into potent lymphostimulatory cells, a process that is associated with up-regulation of costimulatory molecules (CD80, CD86, CD40, DC-lysosomal associated membrane protein (DC-LAMP), CD83), and changes in the chemokine receptors expressed at their surface. Immature DCs, particularly LCs, are CC chemokine receptor (CCR) 6-positive and respond to CC chemokine ligand (CCL) 20 (macrophage inflammatory protein (MIP)-3 $\alpha$ ), whereas mature DCs are attracted by CCL19 (MIP-3 $\beta$ ) or CCL21 (secondary lymphoid chemokine) chemokines following de novo expression of CCR7 [7]. It should be stressed, however, that even these
AFFILIATIONS

*Université Paris 13, UPRES EA-3406, Assistance Publique-Hôpitaux de Paris, Hôpital Avicenne, Laboratoire d'hématologie biologique, Bobigny, \#Université Paris 7, UFR Denis Diderot, Assistance Publique-Hôpitaux de Paris, Service de Pneumologie, Hôpital Saint-Louis, and •INSERM, U-552; Université Paris 7 , UFR Denis Diderot, Paris, France.

\section{CORRESPONDENCE}

A. Tazi

Service de Pneumologie

Hôpital Saint-Louis

1 avenue Claude Vellefaux

75475

Paris cedex 10

France

Fax: 33142499395

E-mail: abdellatif.tazi@sls.ap-hopparis.fr

Received:

October 012005

Accepted after revision:

July 142006

SUPPORT STATEMENT

This work has been supported by a grant from the Ligue contre le Cancer and from the Comité National contre les Maladies Respiratoires.

European Respiratory Journal Print ISSN 0903-1936 Online ISSN 1399-3003 
phenotypically mature DCs can elicit a tolerogenic immune response by preferentially activating regulatory T-cells [8]. Indeed, a critical characteristic of fully mature DCs is the production of pro-inflammatory cytokines, particularly IL-12, which plays a critical role in the induction of an efficient $\mathrm{T}$ helper cell 1 immune response, including antitumour cytotoxic immunity $[3,9]$.

The micro-environment in which DCs are present has an important bearing on the maturation process, and therefore affects the final outcome of the immune response [8]. Thus, IL10 , TGF- $\beta$, vascular endothelial growth factor (VEGF) or prostanoids inhibit the maturation of DCs at different levels, whereas other factors such as LPS, interferon (IFN)- $\gamma$ or early interactions with CD40 ligand-expressing T-cells stimulate their functional capacities $[3,6,8]$.

It is known that tumours avoid surveillance by the immune system through various mechanisms, including the inhibition of the recruitment and/or function of host DCs, and the local production of immunosuppressive factors [10,11]. Thus, the precise knowledge of the tumour micro-environment, which varies between different tumour types, is important for the design of optimal DC-based therapeutic strategies against cancer.

The current authors have previously reported that various numbers of DCs infiltrate lung carcinomas and suggested that these cells were functionally immature [12]. More recently, novel markers have emerged that allow the identification in tissue sections of a broader spectrum of DC subpopulations as well as better assessment of their functional status. In the present study, these new tools have been used to further characterise DC populations present in nonsmall cell lung carcinomas, and to evaluate the local expression of chemokines that may play a role in the recruitment of these cells. Using real-time PCR, the production of factors in the tumour microenvironment that are known to have a critical influence on DC function was also analysed.

\section{METHODS}

\section{Tissue specimens}

Biopsies of primary lung carcinomas were obtained from 12 patients (nine males; three females; mean age $64 \pm 8 \mathrm{yr}$, all smokers) at the time of thoracic surgery. The histological types of cancer were: squamous-cell carcinoma $(n=5)$, adenocarcinoma $(n=5)$; undifferentiated large-cell carcinoma $(n=2)$. No patient had received chemotherapy or radiotherapy at the time of evaluation. The study was approved by the institutional review board of the Hôpital Saint-Louis.

\section{Processing of tissues and morphologic assessment}

Lung-tumour biopsies were immediately snap frozen and stored in liquid nitrogen until required for isolation of RNA or immunohistochemical evaluation. The histopathological features of the frozen specimens were evaluated using cryostat sections stained with haematoxylin end eosin. The entire tumoural surface of each section was quantified using an image analysis system (Microvision; Histolab, Evry, France). In all cases, a second tissue fragment was fixed in BouinHollande solution, processed by routine techniques and used for diagnostic purposes. Two paraffin-embedded specimens were also used for immunostaining with anti-CCR6 antibody.

\section{Isolation of RNA and cDNA synthesis}

Total RNA from each sample was isolated using an Rneasy Mini kit (Qiagen $\mathrm{GmbH}$, Hilden, Germany) according to the manufacturer's instructions and quantified by measurement of absorbance at $260 \mathrm{~nm}$. Complementary DNA (cDNA) was synthesised using an Omniscript reverse transcriptase kit (Qiagen) according to the manufacturer's instructions.

\section{Quantification of cDNAs by real-time PCR}

A $10 \mu \mathrm{L}$ aliquot of each cDNA sample was amplified in the presence of $25 \mu \mathrm{L}$ Taqman universal PCR master mix (PerkinElmer, Foster City, CA, USA), $2.5 \mu \mathrm{L}$ Assays-on-Demand (Perkin-Elmer) containing specific primers and probe for each gene target evaluated (CCL20, CCL19, granulocyte-macrophage colony-stimulating factor (GM-CSF), IL-12, IFN- $\gamma$, IL-10, TGF- $\beta$, IL-4, VEGF) and $22.5 \mu \mathrm{L}$ of water (50 $\mu \mathrm{L}$ final volume). The reaction mixture for amplification of the internal positive control (reduced glyceraldehyde phosphate dehydrogenase) contained $25 \mu \mathrm{L}$ Taqman universal PCR master mix, $10 \mu \mathrm{L}$ cDNA, $200 \mathrm{nM}$ each specific forward (5'-ACC CCT GGC CAA GGT CAT C) and reverse (5'-AGG GGC CAT CCA CAG TCT TC) primers, $200 \mathrm{nM}$ TaqMan probe [5'-(6-carboxyfluorescein (FAM))-AGG ACT CAT GAC CAC AGT CCA TGC CAT (minor groove binder (MGB))] and water (50 $\mu \mathrm{L}$ final volume). In all cases, cycling parameters were as follows: $50^{\circ} \mathrm{C}$ for $2 \mathrm{~min}$, $95^{\circ} \mathrm{C}$ for $10 \mathrm{~min}$, followed by 40 cycles at $95^{\circ} \mathrm{C}$ for $15 \mathrm{~s}$ and $60^{\circ} \mathrm{C}$ for $1 \mathrm{~min}$. Amplifications of the specific cDNA and the internal positive control were always performed at the same time and in parallel for all samples. Gene-specific PCR products were measured by means of an ABI PRISM 7000 Sequence Detection System (Perkin-Elmer). The number of cycles required to reach threshold fluorescence $\left(C_{t}\right)$ was determined, and the quantity of sequences initially present was calculated by extrapolation onto the standard curve. All reactions were performed in duplicate, and the mean of the two values was used for calculations. Target gene expression was normalised between different samples based on the values of the expression of the internal standard.

\section{Immunohistochemical techniques}

Monoclonal antibodies used in this study were: anti-CD11c (BU15; Immunotech, Marseille, France); anti-CD1a (BL6; Immunotech); anti-Langerin (DCGM4; Immunotech); antiCCR6 (53103.111; R\&D Systems, Abingdon, UK); anti-DCLAMP (104.G4; Immunotech); anti-CD83 (HB15a; Immunotech); anti-IL-3R (S-12; Santa Cruz Biotechnology, Santa Cruz, CA, USA); anti-CD3 (UCHT1; Immunotech); anti-CD19 (J4.119; Immunotech). Immunoglobulin (Ig)G1 (679.1Mc7; Immunotech) and IgG2b (MOPC-195; Immunotech) control antibodies were used to assess nonspecific binding. Purified goat polyclonal antibodies used were: anti-MIP-3 $\alpha$ (CCL20); antiMIP-3 $\beta$ (CCL19); anti-DC-specific intercellular adhesion molecule-grabbing nonintegrin (anti-DC-specific intracellular adhesion molecule grabbing nonintergin (SIGN)) and antiCCR7 (all from Santa Cruz).

Immunohistochemical staining was performed as previously described [12], using Vectastain ABC-alkaline phosphatase 
system (Vector, Burlingame, CA, USA) and the fast red substrate. Immunostaining for CCR6 on two paraffinembedded specimens was performed as previously described [13]. The intensity of immunostaining was graded from (absent) to +++ (strongly positive). Complete agreement in scoring was obtained between two independent observers. To determine the numbers of CD1a+ and Langerin+ cells within a given biopsy, positive cells were counted using a light microscope at a magnification of $400 \times(40 \times$ objective and $10 \times$ eyepiece), and the entire surface of serial sections stained with the two antibodies was evaluated. Results are expressed as cells $\cdot \mathrm{mm}^{-2}$ of the tissue section. Inter-observer variation was $<10 \%$.

\section{Statistical analysis}

Results are presented as mean $\pm \mathrm{SD}$, unless otherwise stated. Comparisons were made using the Mann-Whitney U-test, except for histological type and lymph-node staging which were made using the Fisher exact test. Correlation analyses between the number of CD1a+ cells infiltrating tumour specimens and the chemokines and cytokines studied were performed using the Spearman correlation coefficient. A pvalue of $<0.05$ was considered significant.

\section{RESULTS}

\section{Surface phenotype of dendritic cells infiltrating nonsmall cell lung carcinomas}

Various numbers of CD11c+ DCs (i.e. myeloid DCs) were present in all nonsmall cell carcinoma specimens. In contrast, no CD123+ (IL-3R) DCs (i.e. plasmacytoid DCs) were observed in the tumour tissues evaluated in the present study, although some endothelial cells were CD123+, as confirmed by antiCD31 immunostaining on serial sections (not shown).

The lung-tumour specimens were variably infiltrated by CD1a+ DCs. The distribution of these CD1a+ cells was not uniform within a given tumour and positive cells were essentially localised within tumour nodules interspersed among tumour cells (fig. 1a). Interestingly, the 12 samples evaluated could be separated into two groups according to the numbers of CD1a+ cells infiltrating the tissue sections: six samples were heavily infiltrated by CD1a+ DCs, whereas few CD1a+ cells were identified in the remaining six specimens (table $1 ; 18.9 \pm 8.4$ and $2.3 \pm 1.2$ cells $\cdot \mathrm{mm}^{-2}$ respectively; $\mathrm{p}<0.01$ comparing the two groups). The numbers of CD1a+ cells present within a tissue specimen were not correlated with the histological type or the lymph-node staging of the tumour obtained after surgery (table $1 ; \mathrm{p}>0.2$ ). Langerin+ cells were unequivocally observed in several tumour-tissue specimens and had the same distribution as that of CD1a+ cells (fig. 1b). Strikingly, however, on serial tissue sections, the numbers of Langerin+ cells were always lower than those of CD1a+ cells (table 1). Furthermore, few or no Langerin+ cells were noted in five specimens, including two lung tumours heavily infiltrated by CD1a+ cells (table 1 ).

Interestingly, DC-SIGN+ cells were also observed in seven lung tumour biopsies (table 1). On serial tissue sections, these cells were shown to be CD1a- and were localised in areas immediately surrounding the tumour infiltrates (fig. 1c). Furthermore, these DC-SIGN+ cells were, without exception, CD68- (fig. 1d). Conversely, no DC-LAMP+ DC was observed in any of the 12 lung tumours evaluated, although in five tumours, type II pneumocytes adjacent to the tumour nodules reacted strongly with the anti-DC-LAMP antibody (fig. 1e). Few CD83+ DCs were observed in 10 out of 12 tumours, essentially in the connective tissue stroma surrounding the tumour nodules (fig. 1f). These cells had a characteristic dendritic shape and were CD3- and CD19- (not shown).

\section{Expression of chemokines and chemokine receptors in lung carcinomas}

mRNA coding for CCL20 was detected in all tissue specimens, although in variable amounts. Strikingly, the amounts of CCL20 mRNA were quantitatively higher in the six specimens heavily infiltrated by CD1a+ cells compared with those measured in the remaining six samples containing few CD1a+ cells $(14.1 \pm 13.3$ and $2.4 \pm 1.6$ respectively; $\mathrm{p}<0.05$; table 2 and fig. 2). Immunostaining with anti-MIP-3 $\alpha$ antibody demonstrated that tumour cells were the main cell type that expressed CCL20, although the intensity of this expression varied widely from one tumour specimen to another. Tumours that were heavily infiltrated by CD1a+ DCs were strongly positive for CCL20 and were graded from ++ (moderately positive) to +++ (strongly positive) by two independent observers (fig. 3a), whereas five out of six of the biopsies containing few CD1a+ cells were negative (-) for this chemokine and the remaining specimen reacted weakly $(+)$ with anti-CCL20 antibody (fig. 3d and fig. 3e). Furthermore, the number of CD1a+ cells present in tumour specimens and the amounts of mRNA coding for CCL20 were strongly correlated $(\mathrm{r}=0.95 ; \mathrm{p}<0.001)$. Surprisingly, however, CD1a+ DCs present in the tumour specimens were always CCR6 negative, although CCR6+ lymphocytes were clearly present in the same samples (fig. 3b). No CCR6+ DCs were observed in two paraffin-embedded tumour specimens expressing high amounts of CCL20 mRNA (data not shown).

mRNA coding for CCL19 was also detected in all specimens studied. No difference in the level of expression of CCL19 mRNA was observed, however, on comparing tumours heavily infiltrated by CD1a+ cells with the specimens containing few CD1a+ cells $(8.6 \pm 10.6$ and $5.2 \pm 4.7$, respectively; $\mathrm{p}>0.2$; table 2). Immunostaining with anti-CCL19 antibody was performed in 11 lung tumour biopsies and was positive in five specimens (Fig. 4a). Apart from tumour cells, bronchiolar epithelial cells, endothelial cells and some lymphocytes were also positive for this chemokine. No correlation was observed between the CCL19 mRNA expression or immunostaining and the presence of CD83+ DCs or DC-SIGN+ cells. Finally, the only CCR7+ cells present in four tumour specimens were endothelial cells as confirmed by morphological analysis and CD31 immunostaining (fig. 4b).

\section{Cytokine pattern expressed in the tumoural micro- environment}

To evaluate the potential effects of the tumour microenvironment on DCs present in lung carcinomas, real-time PCR was used to measure the expression of mRNAs coding for GM-CSF, IFN- $\gamma$, IL-12, IL-4, IL-10, TGF- $\beta$ and VEGF in the 12 lung tumour biopsies. As previously reported by the current authors, GM-CSF mRNA was detected in all samples, but was present in greater amounts in the tumour biopsies heavily 

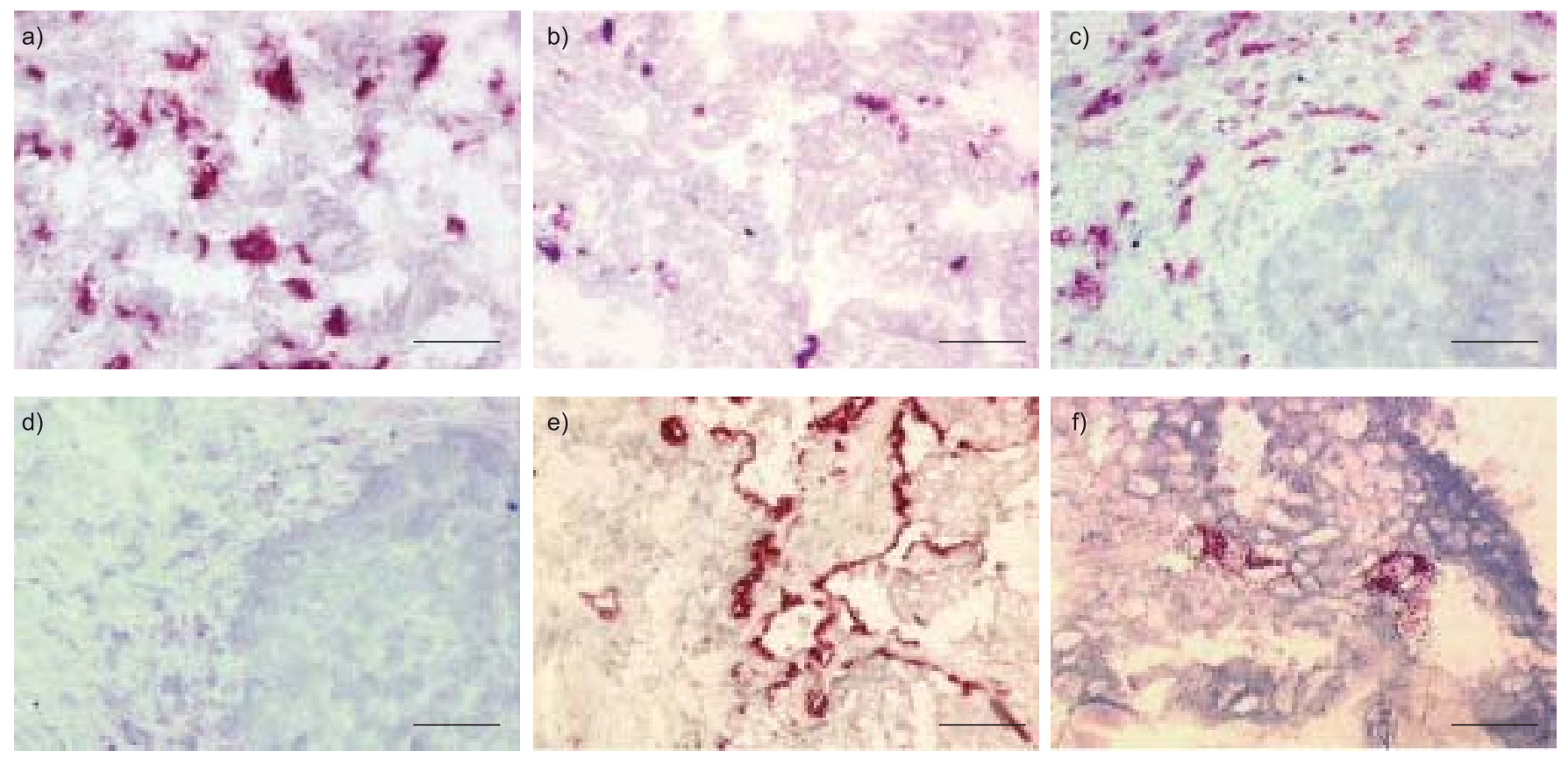

FIGURE 1. Phenotype of dendritic cells (DCs) infiltrating lung carcinomas. a) Adenocarcinoma tumour nodule containing numerous CD1a+ cells. b) Another section of the same tumour showing infiltration by Langerin+ cells. c) A squamous cell carcinoma showing the presence of DC-specific intercellular adhesion molecule-grabbing nonintegrin-positive cells surrounding the tumour nodules. Scale bar $=100 \mu \mathrm{m}$. d) A serial section from the specimen shown in figure $1 \mathrm{c}$ is negative with an anti-CD68 antibody. e) DC-lysosomal associated membrane protein immunostaining. No positive DCs were observed. Note that the epithelial cells adjacent to the tumour nodules are strongly positive. f) Few CD83+ cells are present in this adenocarcinoma. a-d) Scale bars $=100 \mu \mathrm{m}$, e) scale bar $=180 \mu \mathrm{m}$ and f) scale bar=75 $\mu \mathrm{m}$.

\begin{tabular}{llllll}
$\mathbf{T A B L E} \mathbf{1}$ & $\begin{array}{l}\text { Phenotype of dendritic cells infiltrating lung } \\
\text { carcinomas }\end{array}$ & & & \\
Patient & $\begin{array}{c}\text { Histologic } \\
\text { type }\end{array}$ & pTNM $^{\#}$ & CD1a & Langerin & DC-SIGN \\
& ADC & pT2N2 & 28.2 & 0 & - \\
$\mathbf{1}$ & SCC & pT1NO & 27.7 & 10.6 & - \\
$\mathbf{2}$ & ADC & pT3NO & 16.5 & 0.4 & - \\
$\mathbf{3}$ & SCC & pT3N2 & 9 & 6.4 & + \\
$\mathbf{4}$ & ADC & pT4NO & 21.9 & 4.9 & ++ \\
$\mathbf{5}$ & SCC & pT2N2 & 10.3 & 3.8 & ++ \\
$\mathbf{6}$ & ADC & pT2N1 & 2.7 & 0 & ++ \\
$\mathbf{7}$ & LCC & pT3N2 & 2.2 & 0 & + \\
$\mathbf{8}$ & ADC & pT2N1 & 2.9 & 0.6 & + \\
$\mathbf{9}$ & SCC & pT2NO & 1.6 & 0.2 & + \\
$\mathbf{1 0}$ & LCC & pT4N2 & 0.4 & 0 & - \\
$\mathbf{1 1}$ & SCC & pT2NO & 3.9 & 0 & - \\
$\mathbf{1 2}$ & & & & & + \\
\hline
\end{tabular}

Data for CD1a and Langerin are presented as cells· $\mathrm{mm}^{-2}$. DC-SIGN: dendriticcell specific intracellular adhesion molecule grabbing nonintegrin; PTNM: pathological tumour-node-metastasis classification; ADC: adenocarcinoma; SCC: squamous cell carcinoma; LCC: large cell carcinoma; -: absent; ++: numerous. ${ }^{*}:$ p>0.2 comparing histological type and lymph-node staging obtained after surgery $(\mathrm{pN})$ between the specimens containing numerous and few CD1a+ cells, respectively. All patients were MO.

infiltrated by CD1a+ cells $(18.2 \pm 16.6$ and $1.5 \pm 1.6$ copies, respectively; $\mathrm{p}<0.05$; table 2 and fig. 2 ). The number of $\mathrm{CD} 1 \mathrm{a}+$ cells was strongly correlated with the amounts of mRNA coding for GM-CSF expressed in all 12 samples $(\mathrm{r}=0.94$; $\mathrm{p}<0.001$ ). mRNAs coding for VEGF, TGF- $\beta$, IL-10 and IFN- $\gamma$ were expressed in all specimens, regardless of the number and phenotype of DCs present in the tumours (table 2) and no correlation was observed with the number of CD1a+ cells present in tumour specimens $(r<0.3$ and $\mathrm{p}>0.3$ for all cytokines). IL-4 and IL-12 mRNAs were detected in only two out of 12 specimens for each cytokine (table 2). Notably, the expression of IL-4 mRNA was not associated with the presence of DC-SIGN+ DCs in the same tumour biopsies.

\section{DISCUSSION}

The present study has shown that: 1) different subsets of immature myeloid DCs, but not plasmacytoid DCs, infiltrate nonsmall cell carcinomas; 2 ) MIP-3 $\alpha$ (CCL20) produced locally may play a role in the recruitment of CD1a+ cells in these tumours; and 3) lung carcinomas produce several factors that are known to inhibit the function of DCs.

\section{Phenotype of DCs infiltrating lung carcinomas}

All tumour specimens evaluated in the present study contained CD1a+ DCs, although in variable numbers from one tumour to another. As previously reported by the current authors [14], these cells were essentially localised within tumour nodules and interspersed among tumour cells. Presently, it is shown that this population of DCs can be further divided into CD1a+ Langerin+ cells (i.e. LCs) and CD1a+ Langerin- cells. Similar CD1a+ subsets have been also identified in breast carcinomas $[15,16]$. The CD1a+ Langerincells could be precursors of LCs, or the intratumoural counterpart of the inflammatory dendritic epidermal cells described in some skin disorders [6, 17]. 


\begin{tabular}{|c|c|c|c|c|c|c|c|c|c|}
\hline Patient & $\mathrm{CCL}^{\prime \#}{ }^{\#}$ & GM-CSF ${ }^{\#}$ & CCL19 & TGF- $\beta$ & IL-10 & VEGF & IFN- $\gamma$ & IL-4 & IL-12 \\
\hline 2 & 20 & 15 & 8 & 16 & 5 & 19 & 67 & 0 & 0 \\
\hline 3 & 6 & 14 & 2.9 & 11 & 6 & 23 & 221 & 0 & 4 \\
\hline 6 & 4.5 & 6 & 0.5 & 2 & 6 & 3 & 3 & 0 & 0 \\
\hline 7 & 4.2 & 1.1 & 13.6 & 8 & 5 & 15 & 22 & 0 & 0 \\
\hline 8 & 3.3 & 0.6 & 7.7 & 2 & 19 & 3 & 43 & 0 & 0 \\
\hline 9 & 2.1 & 4.7 & 3 & 6 & 7 & 22 & 18 & 0 & 0 \\
\hline 10 & 0.5 & 1 & 1.8 & 5 & 4 & 15 & 62 & 0 & 0 \\
\hline 11 & 0.7 & 0.3 & 1.6 & 14 & 5 & 21 & 57 & 0 & 0 \\
\hline
\end{tabular}

Data obtained by real-time PCR are expressed as number of copies initially present. CCL: CC chemokine ligand; GM-CSF: granulocyte-macrophage colony-stimulating factor; TGF: transforming growth factor; IL: interleukin; VEGF: vascular endothelial growth factor; IFN: interferon. ${ }^{*}: \mathrm{p}<0.05$ comparing specimens containing numerous (patients 1-6) and few (patients 7-12) CD1a+ cells.

Also presently identified is another population of DCs that was CD1a- and expressed DC-SIGN, a C-type lectin involved in capture of antigen and interactions with T-lymphocytes [18]. These cells were present in seven out of 12 lung carcinomas and were always localised at the periphery of the tumour beds. In addition to their dendritic morphology, DC-SIGN+ cells were always CD68-, as assessed using serial tissue sections, further confirming that they were not macrophages. DCSIGN+ DCs were very recently identified in thyroid carcinomas and melanomas, and these cells were also localised at the periphery of the tumours $[19,20]$. Finally, although it has been reported that IL-4 was an important factor for the expression of DC-SIGN [21], mRNA coding for this cytokine was not detected by real-time PCR in the seven specimens that were infiltrated by this DC subset, suggesting that other factors are involved in DC-SIGN expression at the surface of DCs in vivo.

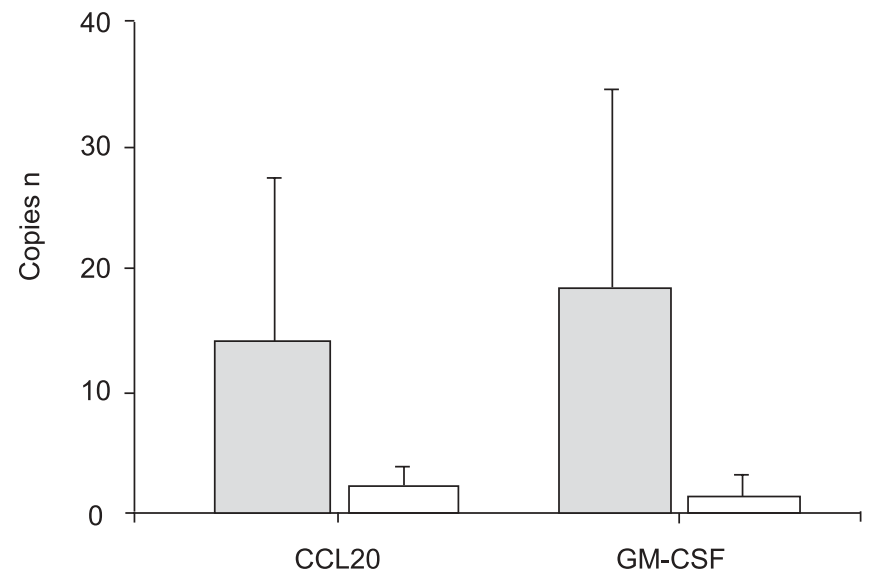

FIGURE 2. The expression of a) CC chemokine ligand (CCL) 20 and b) granulocyte-macrophage colony-stimulating factor (GM-CSF) mRNAs in tumours that were heavily infiltrated by CD1a+ cells $(\square)$ and tumours containing few CD1a+ cells $(\square)$. Results of real-time PCR are expressed as number of copies initially present. $p<0.05$ comparing the two groups of tumours.
The significance of the presence of these DC-SIGN+ DCs in human tumours remains to be studied.

Very few CD83+ DCs were observed in the connective stroma of lung carcinomas, indicating that most of the DC subsets infiltrating these tumours were immature DCs. These cells had a characteristic dendritic shape and were not stained with antiCD3 or anti-CD19 antibodies, further confirming that they were not activated T- or B-lymphocytes. Consistent with this idea, no tumour-infiltrating DC expressed DC-LAMP, another marker of DC maturation. These results are in agreement with previous observations by the current authors that CD1a+ cells in lung cancers were CD40, CD80 and CD86 negative [12]. Strikingly, however, epithelial cells in the peritumoural tissue, particularly type II pneumocytes, expressed DC-LAMP intensely. The expression of DC-LAMP by type II pneumocytes in normal human lung has been reported very recently [22]. Thus, DC-LAMP should no longer be regarded as specific for DCs, as was initially thought.

Plasmacytoid DCs infiltrating other types of carcinomas were recently identified, and it has been suggested that these cells may be involved in the impaired immune response against tumours and adverse outcome [20, 23]. In contrast, plasmacytoid DCs were not identified in the lung carcinomas presently studied. This did not reflect technical problems, since CD123+ endothelial cells (as confirmed by the staining of these cells for the CD31 marker) were shown to be present in the same tissue sections. Interestingly, CONEJO-GARCIA et al. [24] have recently described, in an animal model, a population of DCs that migrate to the endothelium and express markers of both dendritic and endothelial lineage. Thus, although CD123+ cells identified in the current study had a characteristic endothelial shape, the possibilty that these cells represent the counterpart of endothelial DCs can not be excluded.

\section{Chemokine production by lung carcinomas}

In the current study, all tumour specimens expressed mRNA coding for CCL20, but the expression was significantly 

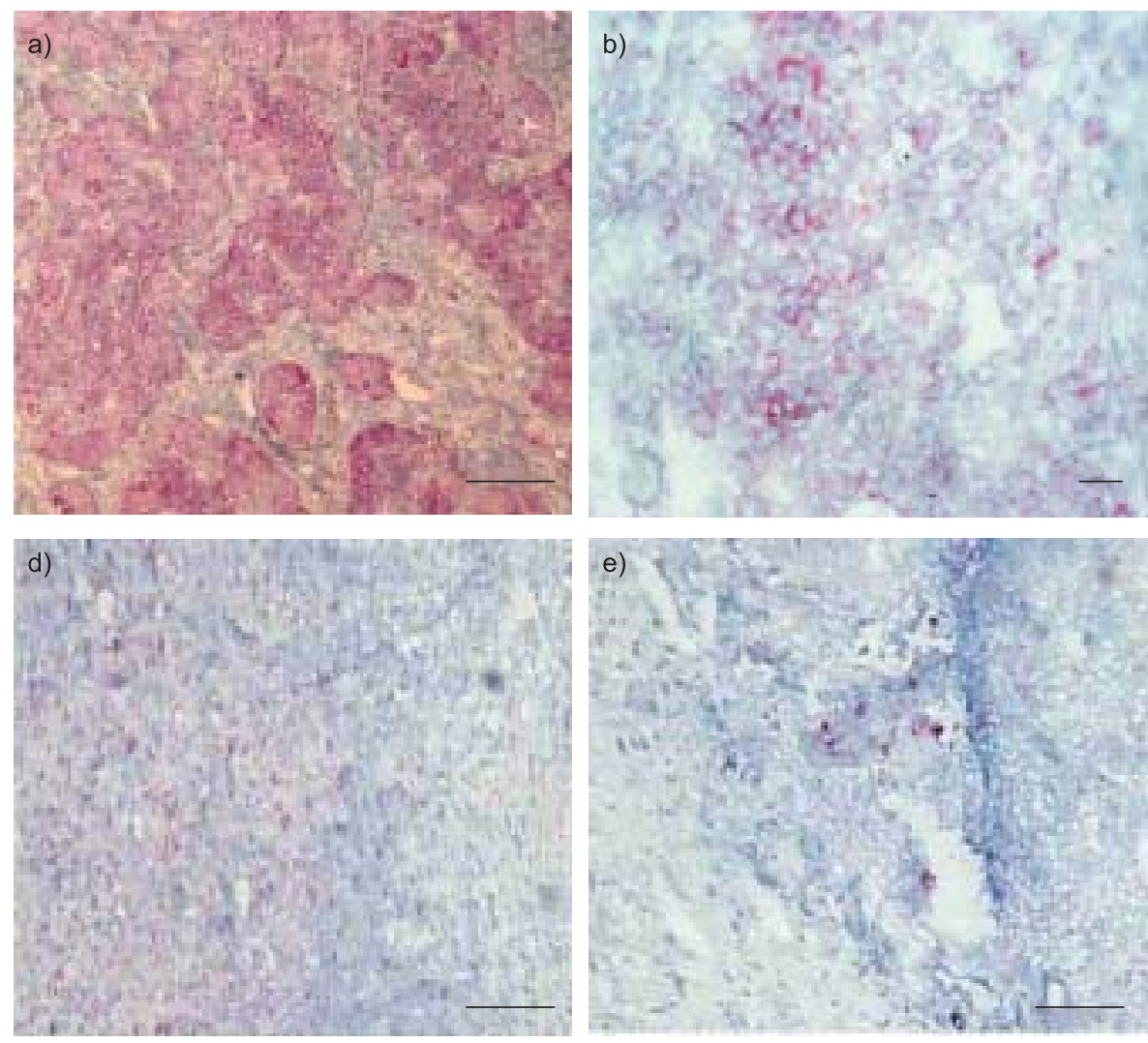

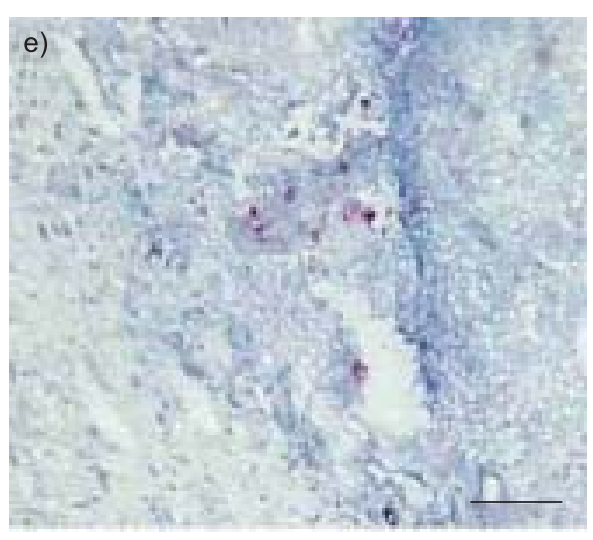

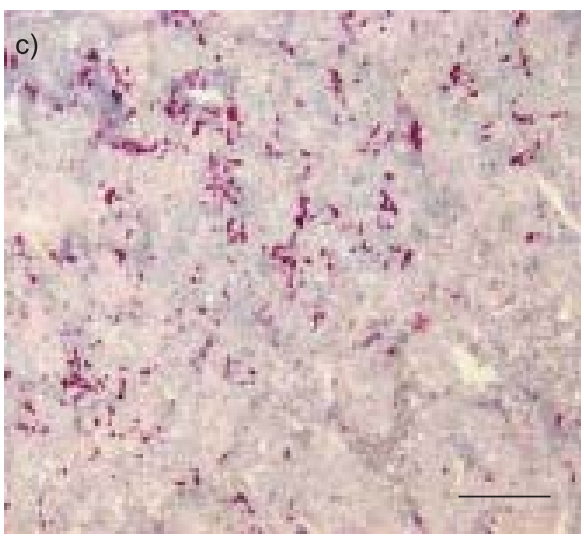

FIGURE 3. Comparison between the production of $C C$ chemokine ligand (CCL) 20 by lung cancers and the presence of CD1a+ cells. a) A squamous-cell carcinoma showing strong positive reaction of tumour cells with anti-CCL20 antibody. b) Although no CC chemokine receptor (CCR) 6-positive dendritic cells were identified, some lymphocytes, i.e. Iymphoid agugates, were CCR6+ in the same sample. c) Serial section of the same tissue specimen containing numerous CD1a+ cells among tumour cells. d) Adenocarcinoma weakly positive for CCL20 immunodetection. e) Few CD1a+ cells were present in serial tissue section from the tumour shown in $d . a, c-e)$ Scale bars $=200 \mu \mathrm{m}$, b) scale bar $=25 \mu \mathrm{m}$. correlated with the number of CD1a+ cells infiltrating lung carcinomas. Evaluation of the expression of this chemokine at the protein level, using immunohistochemistry, showed that tumour cells were the main source of this chemokine, and further confirmed the correlation between the intensity of this expression and the burden of tumour-infiltrating CD1a+ DCs. Expression of CCL20 has also been reported in other tumours, although a correlation with the presence of a particular subset of DCs was not established [16, 19]. Interestingly, in some experimental models, the expression of CCL20 in tumours was associated with the local recruitment of DCs, although the final outcome of the antitumour response was controversial [25-28].

Strikingly, the present study failed to identify CCR6+ DCs in any tumour specimen, despite repeated efforts, whereas CCR6+ lymphocytes were observed in the same tissue sections, suggesting that the expression of CCR6 at the surface of DCs
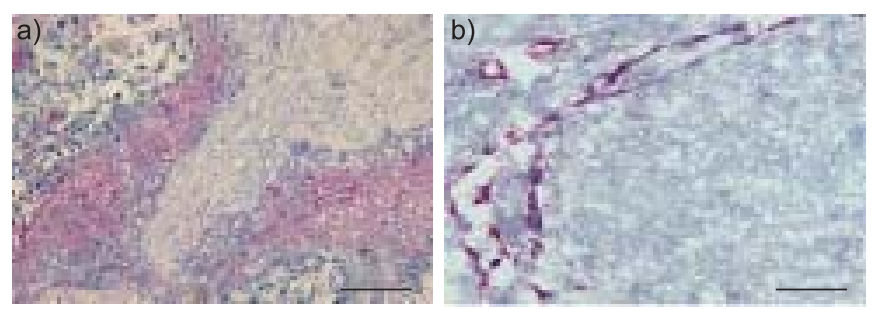

FIGURE 4. a) Immunostaining for CC chemokine ligand 19 in an adenocarcinoma showing positive tumour nodules. Scale bar $=200 \mu \mathrm{m}$. b) A squamous-cell carcinoma stained with anti-CC chemokine receptor 7 antibody. Note that only endothelial cells are positive. Scale bar $=150 \mu \mathrm{m}$. was weak and not detectable by the antibodies used. In this regard, although a correlation between the expression of CCL20 and the infiltration of breast carcinomas by CD1a+ DCs has been reported, the expression of CCR6 receptor by these cells was not evaluated [16]. Similarly, although the in situ expression of CCR6 receptor has been assessed by immunohistochemistry in some pathological tissues, the specimens were paraffin-embedded and antigen-retrieval procedures were performed prior to immunostaining [19, 29]. Indeed, little data are currently available concerning the detection of CCR6 at the surface of DCs in snap-frozen tissues. To further address this point, two paraffin-embedded tumour specimens expressing high amounts of CCL20 mRNA were stained with anti-CCR6 antibody and shown to contain no positive DCs. Thus, the expression of CCR6 antigen at the surface of DCs was probably below the threshold level of detection of the immunohistochemical techniques used.

CCL19 chemokine mRNA was expressed in all lung carcinomas evaluated. In contrast to CCL20 expression, several cell types, apart from tumour cells, were stained with the antiCCL19 antibody. This production of CCL19 is somewhat surprising, since few mature (CD83+) DCs were observed in lung carcinomas, and no correlation could be established between CCL19 and the presence of any subset of DCs present in tissues evaluated, including the number of CD1a+ cells. CCL19 expression may play a role in the recruitment of naïve T-cells or natural killer cells, a hypothesis that was not evaluated in the present study $[7,30]$. It should be noted, however, that the only CCR7+ cell type identified was that of endothelial cells. Thus, additional studies are needed to define 
the role of CCL19 in lung cancer, and to determine whether CCL19 expression is beneficial or detrimental for antitumoural immunity.

\section{Cytokine micro-environment in lung carcinomas}

A previous finding of the current authors, that GM-CSF was expressed in nonsmall lung carcinomas and that the levels of expression correlated with the numbers of CD1a+ infiltrating cells [14], was confirmed by the present study. These findings suggest that CCL20 is not the only mediator involved in the recruitment of DCs in lung cancers. Consistent with the immature phenotype of DCs infiltrating lung carcinomas, the balance of the cytokine pattern in the tumour micro-environment was, however, clearly shifted toward an inhibitory milieu for DCs maturation. Thus, in all specimens, mRNAs coding for IL-10, TGF- $\beta$ and VEGF were detected, regardless of the subsets of DCs present in the tumour, and no correlation was observed with the number of CD1a+ cells. These three factors are potent inhibitory mediators of DC maturation and function, and have been associated to various extents with a defective antitumour immune response [10, 11, 31, 32]. In contrast, although IFN- $\gamma$ mRNA was present in all tumours, only two samples contained mRNA coding for IL-12, a critical cytokine for mounting efficient cytotoxic antitumour immune responses both in animal models and in human trials [3, 9]. Taken together, the virtual absence of IL-12 and the predominance of inhibitory factors of DC maturation suggest that the tumour micro-environment is unlikely to be propitious for T-cell activation.

The present study is limited, however, by the small number of specimens evaluated and results obtained here, although clear cut, should be further confirmed.

Nevertheless, these results shade new light on dendritic-cell biology in lung cancers, and show that tumour microenvironment is essentially inhibitory of dendritic-cell maturation. These results should be considered when elaborating strategies using dendritic cell-based immunotherapy for patients with lung cancers.

\section{ACKNOWLEDGEMENTS}

The authors would like to thank M. Mao for excellent technical assistance.

\section{REFERENCES}

1 Nestle FO, Alijagic S, Gilliet M, et al. Vaccination of melanoma patients with peptide- or tumor lysate-pulsed dendritic cells. Nat Med 1998; 4: 328-332.

2 Kugler A, Stuhler G, Walden P, et al. Regression of human metastatic renal cell carcinoma after vaccination with tumor cell-dendritic cell hybrids. Nat Med 2000; 6: 332-336.

3 Banchereau J, Palucka AK. Dendritic cells as therapeutic vaccines against cancer. Nat Rev Immunol 2005; 5: 296-306.

4 Ishikawa A, Motohashi S, Ishikawa E, et al. A phase I study of alpha-galactosylceramide (KRN7000)-pulsed dendritic cells in patients with advanced and recurrent non-small cell lung cancer. Clin Cancer Res 2005; 11: 1910-1917.

5 Hirschowitz EA, Foody T, Kryscio R, Dickson L, Sturgill J, Yannelli J. Autologous dendritic cell vaccines for nonsmall-cell lung cancer. J Clin Oncol 2004; 22: 2808-2815.
6 Ardavin C, Martinez del Hoyo G, Martin P, et al. Origin and differentiation of dendritic cells. Trends Immunol 2001; 22: 691-700.

7 Sallusto F, Lanzavecchia A. Understanding dendritic cell and T-lymphocyte traffic through the analysis of chemokine receptor expression. Immunol Rev 2000; 177: 134-140.

8 Steinman RM, Hawiger D, Nussenzweig MC. Tolerogenic dendritic cells. Annu Rev Immunol 2003; 21: 685-711.

$9 \mathrm{Xu} \mathrm{S,} \mathrm{Koski} \mathrm{GK,} \mathrm{Faries} \mathrm{M,} \mathrm{et} \mathrm{al.} \mathrm{Rapid} \mathrm{high} \mathrm{efficiency}$ sensitization of CD8+ $\mathrm{T}$ cells to tumor antigens by dendritic cells leads to enhanced functional avidity and direct tumor recognition through an IL-12-dependent mechanism. J Immunol 2003; 171: 2251-2261.

10 Zou W. Immunosuppressive networks in the tumour environment and their therapeutic relevance. Nat Rev Cancer 2005; 5: 263-274.

11 Yang L, Carbone DP. Tumor-host immune interactions and dendritic cell dysfunction. Adv Cancer Res 2004; 92: 13-27.

12 Tazi A, Moreau J, Bergeron A, Dominique S, Hance AJ, Soler P. Evidence that Langerhans cells in adult pulmonary Langerhans cell histiocytosis are mature dendritic cells: importance of the cytokine microenvironment. J Immunol 1999; 163: 3511-3515.

13 Movassagh M, Spatz A, Davoust J, et al. Selective accumulation of mature DC-Lamp+ dendritic cells in tumor sites is associated with efficient T-cell-mediated antitumor response and control of metastatic dissemination in melanoma. Cancer Res 2004; 64: 2192-2198.

14 Tazi A, Bouchonnet F, Grandsaigne M, Boumsell L, Hance AJ, Soler P. Evidence that granulocyte macrophagecolony-stimulating factor regulates the distribution and differentiated state of dendritic cells/Langerhans cells in human lung and lung cancers. J Clin Invest 1993; 91: 566576.

15 Thomachot MC, Bendriss-Vermare N, Massacrier C, et al. Breast carcinoma cells promote the differentiation of CD34+ progenitors towards 2 different subpopulations of dendritic cells with $\mathrm{CD} 1 \mathrm{a}^{\text {high }}{ }^{\mathrm{CD}} 86^{-}$Langerin $^{-}$and $\mathrm{CD}_{1}{ }^{+} \mathrm{CD} 86^{+}$Langerin $^{+}$phenotypes. Int J Cancer 2004; 110: 710-720.

16 Bell D, Chomarat P, Broyles D, et al. In breast carcinoma tissue, immature dendritic cells reside within the tumor, whereas mature dendritic cells are located in peritumoral areas. J Exp Med 1999; 190: 1417-1426.

17 Wollenberg A, Kraft S, Hanau D, Bieber T. Immunomorphological and ultrastructural characterization of Langerhans cells and a novel, inflammatory dendritic epidermal cell (IDEC) population in lesional skin of atopic eczema. J Invest Dermatol 1996; 106: 446-453.

18 Soilleux EJ. DC-SIGN (dendritic cell-specific ICAM-grabbing non-integrin) and DC-SIGN-related (DC-SIGNR): friend or foe? Clin Sci (Lond) 2003; 104: 437-446.

19 Tsuge K, Takeda H, Kawada S, Maeda K, Yamakawa M. Characterization of dendritic cells in differentiated thyroid cancer. J Pathol 2005; 205: 565-576.

20 Vermi W, Bonecchi R, Facchetti F, et al. Recruitment of immature plasmacytoid dendritic cells (plasmacytoid monocytes) and myeloid dendritic cells in primary cutaneous melanomas. J Pathol 2003; 200: 255-268.

21 Relloso M, Puig-Kroger A, Pello OM, et al. DC-SIGN (CD209) expression is IL-4 dependent and is negatively 
regulated by IFN, TGF- $\beta$, and anti-inflammatory agents. $J$ Immunol 2002; 168: 2634-2643.

22 Salaun B, de Saint-Vis B, Pacheco N, et al. CD208/dendritic cell-lysosomal associated membrane protein is a marker of normal and transformed type II pneumocytes. Am J Pathol 2004; 164: 861-871.

23 Mohty M, Olive D, Gaugler B. Plasmacytoid DCs and cancer: a new role for an enigmatic cell. Trends Immunol 2004; 25: 397-398.

24 Conejo-Garcia JR, Benencia F, Courreges MC, et al. Tumorinfiltrating dendritic cell precursors recruited by a $\beta$ defensin contribute to vasculogenesis under the influence of Vegf-A. Nat Med 2004; 10: 950-958.

25 Bonnotte B, Crittenden M, Larmonier N, Gough M, Vile RG. MIP-3 $\alpha$ transfection into a rodent tumor cell line increases intratumoral dendritic cell infiltration but enhances (facilitates) tumor growth and decreases immunogenicity. J Immunol 2004; 173: 4929-4935.

26 Crittenden M, Gough M, Harrington K, Olivier K, Thompson J, Vile RG. Expression of inflammatory chemokines combined with local tumor destruction enhances tumor regression and long-term immunity. Cancer Res 2003; 63: 5505-5512.

27 Furumoto K, Soares L, Engleman EG, Merad M. Induction of potent antitumor immunity by in situ targeting of intratumoral DCs. J Clin Invest 2004; 113: 774-783.

28 Fushimi T, Kojima A, Moore MA, Crystal RG. Macrophage inflammatory protein $3 \alpha$ transgene attracts dendritic cells to established murine tumors and suppresses tumor growth. J Clin Invest 2000; 105: 1383-1393.

29 Fleming MD, Pinkus JL, Fournier MV, et al. Coincident expression of the chemokine receptors CCR6 and CCR7 by pathologic Langerhans cells in Langerhans cell histiocytosis. Blood 2003; 101: 2473-2475.

30 Vicari AP, Treilleux I, Lebecque S. Regulation of the trafficking of tumour-infiltrating dendritic cells by chemokines. Semin Cancer Biol 2004; 14: 161-169.

$31 \mathrm{Ohm}$ JE, Carbone DP. VEGF as a mediator of tumorassociated immunodeficiency. Immunol Res 2001; 23: 263-272.

32 Sharma S, Stolina M, Lin Y, et al. T cell-derived IL-10 promotes lung cancer growth by suppressing both $\mathrm{T}$ cell and APC function. J Immunol 1999; 163: 5020-5028. 